\title{
COLLECTIVE ION DRAG FORCE
}

\author{
A.M. Ignatov \\ Prokhorov General Physics Institute of the Russian Academy of Sciences, Moscow, Russia
}

We analyse the forces acting upon a set of dust grains aligned perpendicular to a plasma flow. It is shown that there appears the force component which is parallel to the flow velocity. Since the effect arises due to the momentum exchange between an ensemble of dust grains and the ambient plasma, it is called the collective ion drag.

Implementing a simple model, we evaluate the force between two grains and the force exerted upon the hexagonal grid of particles. For the case of a grain ensemble levitating in a plasma sheath, the collective ion drag force is unexpectedly large. It may be comparable or even exceed the weight of single grain. 